\title{
Famílias de grafos com contorno não geodésico
}

\author{
Simone Dantas ${ }^{1, *} \quad \underline{\text { Thiago de M. D. e Silva }}{ }^{2, * *}$ \\ Instituto de Matemática e Estatística \\ Universidade Federal Fluminense \\ 24020-140, Niterói, RJ \\ E-mail ${ }^{1}$ : sdantas@im.uff.br \\ Email ${ }^{2}$ : thiagoomenez@gmail.com
}

\author{
Danilo Artigas* \\ Instituto de Ciência e Tecnologia \\ Universidade Federal Fluminense \\ 28895-532, Rio das Ostras, RJ \\ E-mail: daniloartigas@puro.uff.br
}

\section{RESUMO}

Este trabalho contempla o estudo de conceitos de matemática contínua que podem ser estendidos para matemática discreta, em particular, teoria dos grafos. Um desses conceitos é o de convexidade geodésica.

Seja $G$ um grafo, onde $V(G)$ é o conjunto de vértices de $G$ e $E(G)$ é o conjunto de arestas de $G$. A distância entre dois vértices $u$ e $v$ de $G$ é o número de arestas contidas em um caminho mínimo entre eles. A excentricidade de um vértice $u$ de $G$ é a maior distância entre $u$ e qualquer outro vértice $w$ de $G$. Um intervalo fechado entre dois vértices $u$ e $v$ de $G$, denotado por $I[u, v]$, é um subconjunto de $V(G)$ formado por $u$ e $v$ e todos os vértices que se encontram em qualquer caminho mínimo entre $u$ e $v$. Dado um subconjunto $S$ de $V(G)$, dizemos que $S$ é convexo se $I[S]$ $=S$. Dizemos que $S$ é geodésico se $I[S]=V(G)$. O contorno de um grafo, $C t(G)$, é formado pelos vértices cuja excentricidade é maior ou igual a de seus vizinhos.

O objetivo deste trabalho é estudar sobre quais condições o contorno é um subconjunto geodésico de $G$, e contribuir para o estimulante problema em aberto, proposto em 2005 [2], que consiste na seguinte pergunta: " $I^{2}[C t(G)]=V(G)$, para todo $G$ ?".

O primeiro grafo cujo contorno não era geodésico foi exibido em 2005 [2]. Uma variação deste mesmo grafo foi apresentada em [3] (veja Figura 1). Tendo em vista a dificuldade da determinação dos vértices que pertencem a $C t(G)$, pois a remoção ou inserção de uma aresta ou vértice pode alterar as excentricidades de todos os vértices de $G$, somente em 2013 [1], foi exposto outro grafo cujo contorno não era geodésico, exibido na Figura 2. Na Figura 1 os vértices $a, b$ e $c$ pertencem a $C t(G)$ e na Figura 2, os vértices destacados com um retângulo pertencem a $C t(G)$ :

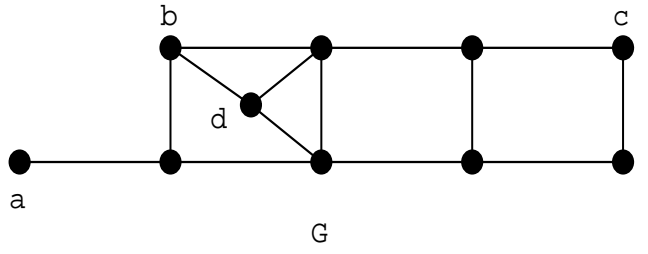

Figura 1: vértice d não pertence à $I[C t(G)]$

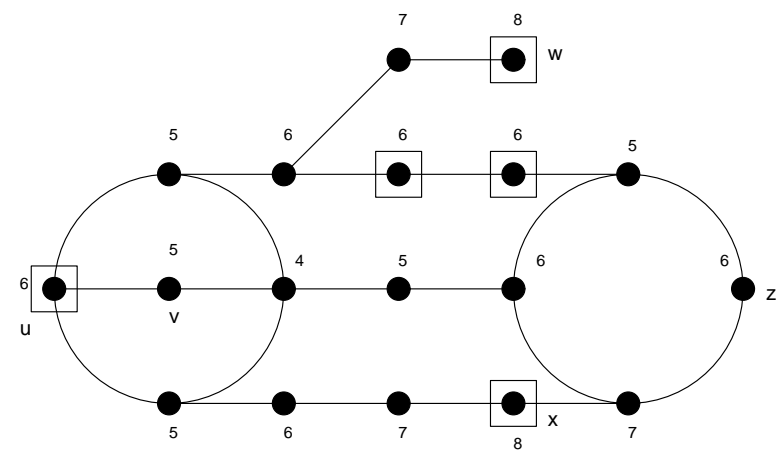

Figura 2: vértice v não pertence à $I / C t(G)]$

Neste trabalho, contribuímos para o estudo deste problema apresentando generalizações de dois exemplos encontrados na literatura. Construímos três famílias infinitas de grafos cujo $C t(G)$ não é geodésico, e duas delas são mostradas a seguir: 


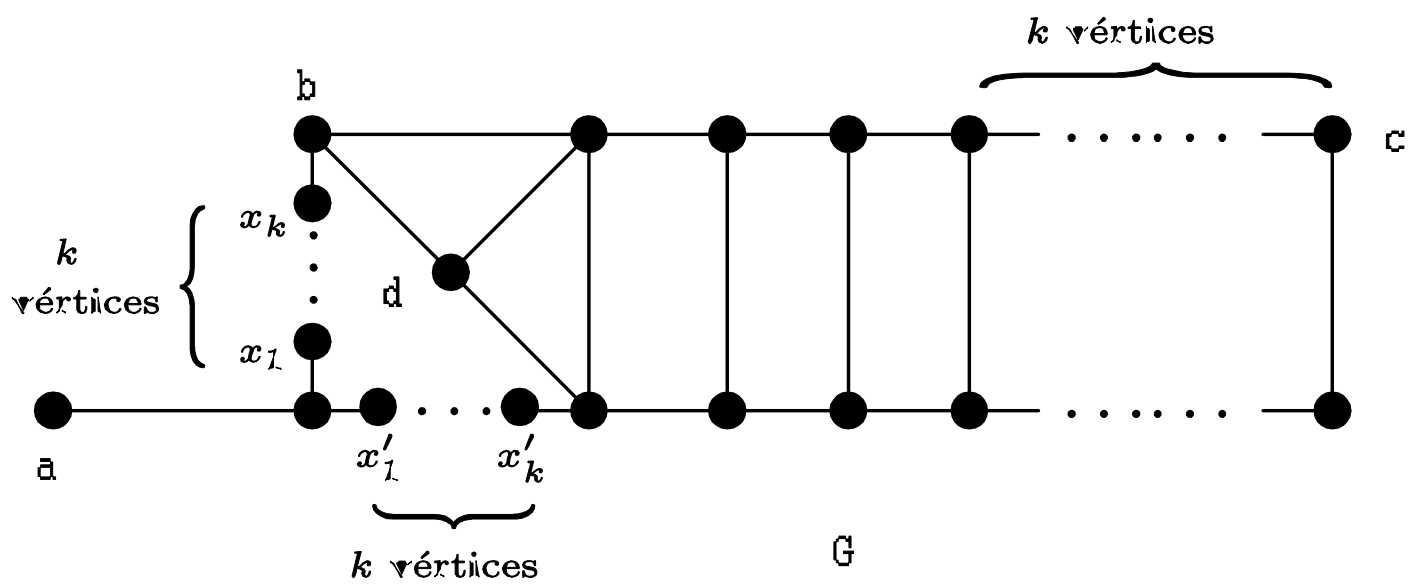

Figura 3: vértice d não pertence a $[[C t(G)]$

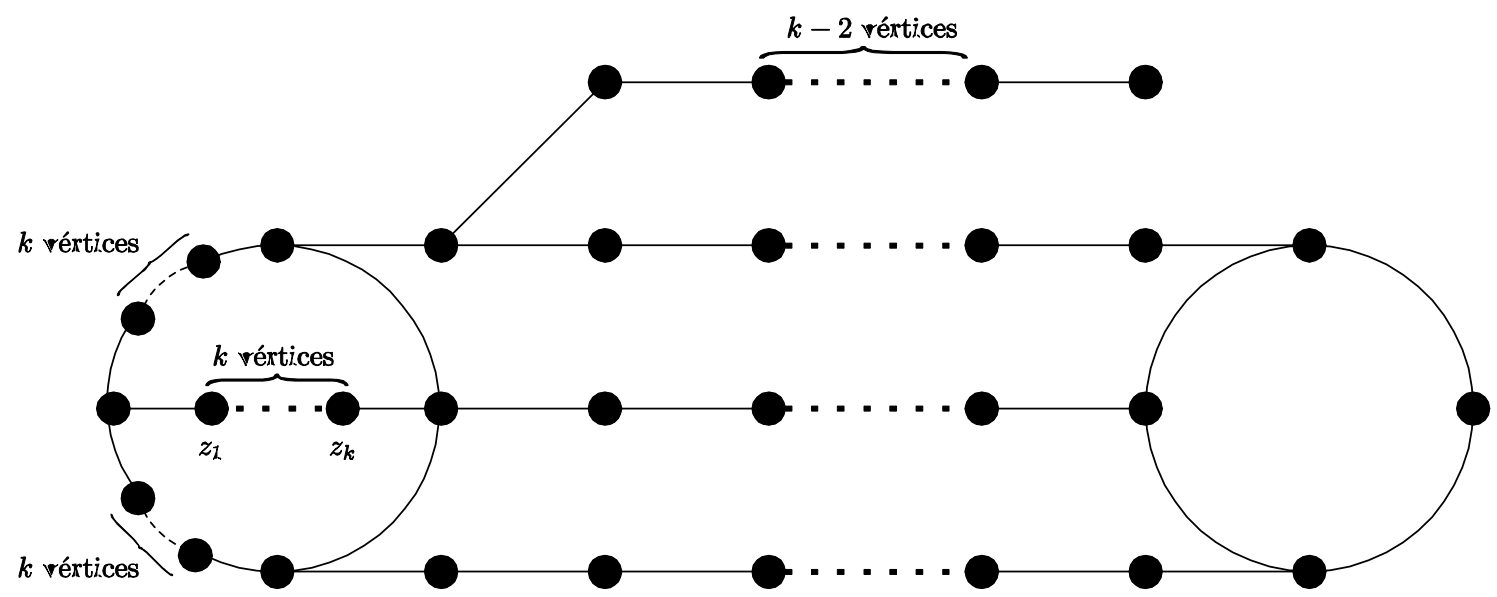

Figura 4: vértices $z_{i}, i \in\{1, \ldots, k\}$, não pertencem a $I[C t(G)]$

A terceira família pode ser obtida através da Figura 3, a partir da remoção de um vértice $x_{i}$ ou de um vértice $x_{i}^{\prime}, i \in\{1, \ldots, k\}$. Da mesma forma, $d$ não pertencerá à $I[C t(G)]$. A prova dessas duas generalizações pode ser feita utilizando-se o princípio de indução matemática.

Até o momento, a única propriedade conhecida é a seguinte: se um conjunto $\mathrm{S}$ de vértices de $G$ é tal que $C t(G) \subseteq S$ e $|S| \geq n$-3 então $S$ é geodésico. Os poucos exemplos apresentados na literatura possuem $|V(G) \backslash I[C t(G)]|=1$ e portanto $I[I[C t(G)]]$ é geodésico. Observamos que na Figura 4, apresentamos uma família infinita de grafos onde $k$ vértices não pertencem ao primeiro intervalo do contorno. Logo, este é o primeiro exemplo onde $\mid \operatorname{ICt}(G)] \mid<n-3$ e $I[C t(G)]$ geodésico.

Palavras-chave: Teoria dos grafos, convexidade em grafos, conjunto de contorno.

\section{Referências}

[1] D. Artigas, S. Dantas, M. C. Dourado, J. L. Szwarcfiter, S. Yamaguchi, On the contour of a graph, Discrete Applied Mathematics, vol. 161, pp. 1356-1362, (2013).

[2] J. Cáceres, A. Márquez, O. R. Oellermann, M. L. Puertas, Rebuilding convex sets in graphs, Discrete Mathematics, vol. 297, pp. 26-37, (2005).

[3] J. Cáceres, C. Hernando, M. Mora, I. M. Pelayo, M. L. Puertas, C. Seara, Geodecity of the contour of chordal graphs, Discrete applied Mathematics, vol. 156, pp. 1132-1142, (2008). 